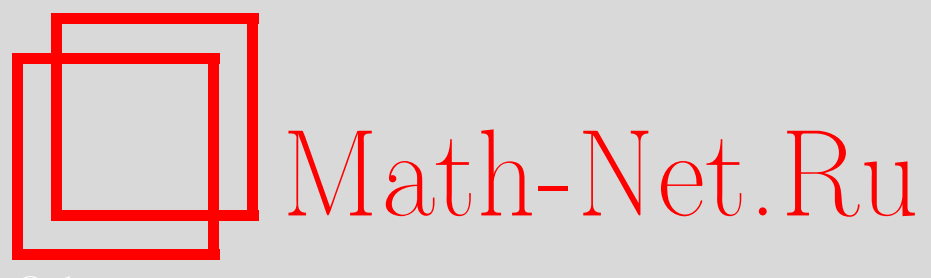

Д. А. Медведев, Асимптотическое поведение решений уравнений с последействием в гильбертовом пространстве, УМН, 2007, том 62, выпуск 1, 205-206

DOI: https://doi.org/10.4213/rm5585

Использование Общероссийского математического портала Math-Net.Ru подразумевает, что вы прочитали и согласны с пользовательским соглашением http://www.mathnet.ru/rus/agreement

Параметры загрузки:

IP : 3.95 .254 .165

26 апреля 2023 г., 14:34:42

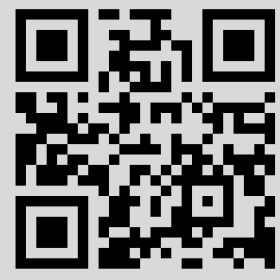




\section{Асимптотическое поведение решений уравнений с последействием в гильбертовом пространстве}

\section{Д. А. Медведев}

В предлагаемой статье приводятся результаты об асимптотическом поведении и неулучшаемых оценках решений неоднородных дифференциально-разностных уравнений, коэффициентами которых являются неограниченные операторы в гильбертовом пространстве.

Обозначим через $L_{2}(a, b),-\infty<a<b \leqslant+\infty$, пространство вектор-функций со значениями в гильбертовом пространстве $H$ с нормой $\|\varphi\|_{L_{2}(a, b)}^{2}=\int_{a}^{b}\|\varphi(t)\|_{H}^{2} d t$, и через $W_{2}^{1}(a, b ; A)$ - пространство Соболева вектор-функций со значениями в $H$ (подробнее см. [1; гл. 1]) с нормой $\|\varphi\|_{W_{2}^{1}(a, b ; A)}^{2}=\|A \varphi\|_{L_{2}(a, b)}^{2}+\left\|\varphi^{\prime}\right\|_{L_{2}(a, b)}^{2}$.

Рассмотрим традиционную начальную задачу для дифференциально-разностного уравнения вида

$$
\begin{gathered}
u^{\prime}(t)+A u(t)+\sum_{k=1}^{n} B_{k} A u\left(t-h_{k}\right)+\int_{0}^{h} B(s) A u(t-s) d s=f(t), \quad t>0, \\
u(s)=g(s), \quad s \in[-h, 0] .
\end{gathered}
$$

Здесь $A$ - положительный самосопряженный оператор, действующий в гильбертовом пространстве $H$ и имеющий компактный обратный. Операторы $B k, B(s)$ имеют вид $B_{k}=T_{k} A^{-\theta_{k}}, B(s)=T(s) A^{-\theta_{0}}$, где $T_{k}$ - ограниченные операторы, $T(s)$ - ограниченная сильно непрерывная оператор-функция. Постоянные $\theta_{k}$ и $h_{k}$ таковы, что $0<\theta_{k} \leqslant 1,0<h_{1}<h_{2}<\cdots<h_{n}=h$.

Обозначим через $L(\lambda)$ характеристическую оператор-функцию уравнения (1):

$$
L(\lambda)=\lambda I+A+\sum_{k=1}^{n} e^{-\lambda h_{k}} B_{k} A+\int_{0}^{h} e^{-\lambda s} B(s) A d s,
$$

где $I$ - тождественный оператор в $H$. Из леммы М. В. Келдыша [2] следует, что $L^{-1}(\lambda)$ - конечномероморфная оператор-функция с дискретным спектром. Обозначим через $\lambda_{q}$ собственные значения $L(\lambda)$, упорядоченные в порядке возрастания модулей с учетом кратности $\nu_{q}$, через $\Lambda$ - множество всех собственных значений оператор-функции $L(\lambda)$.

Из работы [3], в которой изучается поведение в комплексной плоскости характеристической оператор-функции $L(\lambda)$ уравнения (1) и устанавливаются оценки $L^{-1}(\lambda)$, следует, что конечны величины $\varkappa_{+}=\sup _{\lambda_{q} \in \Lambda} \operatorname{Re} \lambda_{q}, \nu_{+}=\max _{\lambda_{q} \in \Lambda}, \operatorname{Re} \lambda_{q}=\varkappa_{+} \nu_{q}$.

Собственные векторы, входящие в каноническую систему [2] собственных и присоединенных векторов оператор-функции $L(\lambda)$, отвечающие числу $\lambda_{q}$, обозначим через $x_{q, j, 0}\left(j=1,2, \ldots, r_{q}\right)$, их присоединенные порядка $s$ - через $x_{q, j, s}\left(s=1,2, \ldots, p_{q j}\right)$. Введем систему экспоненциальных решений однородного уравнения (1):

$$
y_{q, j, s}(t)=e^{\lambda_{q} t}\left(\frac{t^{s}}{s !} x_{q, j, 0}+\frac{t^{s-1}}{(s-1) !} x_{q, j, 1}+\cdots+x_{q, j, s}\right) .
$$

ОпРедЕлЕниЕ. Функцию $u$, принадлежащую пространству $W_{2}^{1}(-h, T ; A)$ для любого $T>0$, назовем сильным решением задачи (1), (2), если и удовлетворяет почти всюду на полуоси $\mathbb{R}_{+}$уравнению (1) и условию $(2)$.

Работа выполнена при поддержке РФФИ (проект № 05-01-00989) и НШ-5247.2006.1. 
Теорема 1. Пусть $g \in W_{2}^{1}(-h, 0 ; A), f \equiv 0 u \beta<\varkappa_{+}$таково, что прямая $\operatorname{Re} \lambda=\beta$ не содержит собственных значений функиии $L(\lambda)$. Тогда для сильного решения и задачи (1), (2) справедливо разложение

$$
u(t)=\sum_{\lambda_{q} \in \Lambda, \operatorname{Re} \lambda_{q}>\beta} c_{q, j, s} y_{q, j, s}(t)+u_{\beta}(t), \quad t>0, \quad u_{\beta} \in W_{2, \beta}^{1}(0,+\infty ; A) .
$$

Tеорема 2. Пустъ $g \in W_{2}^{1}(-h, 0 ; A)$ u $f \in L_{2}(0, T)$ для любых $T>0$. Тогда сильное решение и задачи (1), (2) допускает оченку

$$
\begin{aligned}
\|u\|_{W_{2}^{1}(t-h, t ; A)} \leqslant & d_{1}(t+1)^{\nu_{+}-1} e^{\varkappa+t}\|g\|_{W_{2}^{1}(-h, 0 ; A)} \\
& +d_{2} \sqrt{t}\left(\int_{0}^{t}(t-s+1)^{2\left(\nu_{+}-1\right)} e^{2 \varkappa_{+}(t-s)}\|f(s)\|_{H}^{2} d s\right)^{1 / 2}, \quad t \geqslant 0,
\end{aligned}
$$

где постоянные $d_{1} u d_{2}$ не зависят от функций $g$ u $f$.

ЗАмечАниЕ. В силу локализации спектра $L(\lambda)$ первое слагаемое в правой части разложения (3) содержит лишь конечное число слагаемых. Оценка (4) неулучшаема в том смысле, что величину $\varkappa_{+}$нельзя заменить на $\varkappa_{+}-\varepsilon$ для любого $\varepsilon>0$.

Приведенные результаты справедливы и для конечномерных гильбертовых пространств. Более того, в случае $H=\mathbb{C}^{r}$ результаты теорем 1 и 2 справедливы и для уравнения более общего вида:

$$
u^{\prime}(t)=\int_{-h}^{0} d \mu_{M}(s) u(t+s)+f(t), \quad t>0,
$$

где $\mu_{M}-$ матрица-функция ограниченной вариации, заданная на отрезке $[-h, 0]$. При этом величины $\varkappa_{+}$и $\nu_{+}$определяются точно так же для характеристической матрицыфункции $L(\lambda)$ уравнения (5).

Отметим, что предлагаемый подход в понимании решений и их асимптотического поведения, разумеется, не является единственно возможным. В настоящее время имеется обширная библиография (в основном относящаяся к случаю конечномерного $H)$, в которой представлены различные интерпретации решений и методы изучения начально-краевых задач для функционально-дифференциальных уравнений. Ограничимся указанием статей [4]-[6], относящихся к случаю уравнений в банаховых и, в частности, гильбертовых пространствах.

Автор глубоко признателен В.В.Власову и А. Г. Костюченко за полезные обсуждения работы и ценные предложения и замечания.

\section{Список литературы}

[1] Ж. Л. Лионс, Э. Мадженес, Неоднородные граничные задачи и их приложения, Мир, М., 1971. [2] М. В. Келдыш, УМН, 26:4 (1971), 15-41. [3] В. В. Власов, Матем. сб., 186:8 (1995), 67-92. [4] G. Di Blasio, K. Kunisch, E. Sinestrari, J. Math. Anal. Appl., 102:1 (1984), 38-57. [5] F. Kappel, K. Kunisch, Trans. Amer. Math. Soc., 304:1 (1987), 1-51. [6] K. Kunisch, M. Mastinšek, Differential Integral Equations, 3:4 (1990), 733-756.

Д. А. Медведев (D. A. Medvedev)

Московский государственный университет им. М. В. Ломоносова

E-mail: plop@mail.ru
Представлено А. Г. Костюченко Принято редколлегией 02.11 .2006 